\title{
Extreme Stretching of High G:D Ratio Carbon Nanotube Fibers Using Super-Acid
}

John S. Bulmer, ${ }^{1}$ Jenifer E. Mizen ${ }^{2}$, Thurid S. Gspann², Adarsh Kaniyoor ${ }^{2}$, James B. Ryley ${ }^{1}$, Patrick J. Kiley ${ }^{1,2}$, Martin R. Sparkes, ${ }^{1}$ Bill O'Neill, ${ }^{1}$ James A. Elliott ${ }^{2}$

${ }^{1}$ Institute for Manufacturing, 17 Charles Babbage Road, University of Cambridge, Cambridge, CB3 OFS, United Kingdom

2Department of Materials Science and Metallurgy, 27 Charles Babbage Road, University of Cambridge, Cambridge, CB3 OFS United Kingdom

\begin{abstract}
Few-wall carbon nanotube (CNT) textiles with unparalleled graphitic perfection and a solitary, prominent radial breathing mode (RBM) associated with metallic chirality have been mechanically stretched in chlorosulfonic acid (CSA) to a degree so far unseen in CNT textiles (150 to 250\% of original length) with notably little tension required. This dramatically enhanced their microstructural alignment and density and, after most of the residual CSA was removed by a vacuum bake, the de-doped fiber's electrical conductivity was found to be $45 \%$ greater than single-crystal graphite - a significant milestone for CNT conductor development towards graphitic intercalation compounds (GICs) and traditional metals. Correlation tables and validated, multivariate statistical models show that conductivity is overwhelmingly linked to stretching degree, although eventually saturates near single-crystal graphite levels, implying the existence of a maximum undoped conductivity. The degree of stretching within CSA is correlated with the original mechanical properties (tenacity, elongation-to-break, and linear density); the Raman $G^{\prime}: G$ ratio and the upper-end oxidation temperature in thermogravimetric analysis also predict the best results. Less graphenically pristine CNT materials stretch to a lower degree in CSA, similar to previous reports. This study highlights the importance of post-synthesis processing to achieve superior performance in carbon nanotube textile materials.
\end{abstract}


Electrical cables assembled from carbon nanotubes (CNTs) are $\mathrm{sp}^{2}$ carbon-based bulk conductors with electronic transport confined in less than three dimensions. This resembles older $\mathrm{sp}^{2}$ carbon bulk morphologies where the dimensionality of electronic transport is also confined, such as graphite intercalation compounds (GICS) and iodine-doped polyacetylene (IPAC). GICs are typically highly crystalline graphite or carbon fiber where electron-accepting chemical species reside between graphene planes in ordered stacked stages; the highest reported room temperature conductivities reached $150 \%$ of copper [1] [2] [3] [4] [5] [6]. IPAC is an assembly of one-dimensional conjugated carbon chains with electron-accepting iodine residing between chains [7] [8] [9]; the highest conductivity IPACs reached 25\% of copper's conductivity. CNT cables share sometimes opposing features of either GICs and IPACs. Like GICs for example, they have a non-negligible conductivity before doping that increases only several times after doping [1]; IPAC is initially insulating and, after doping, its conductivity can increase by over a billion times in some cases [9]. CNTs, like IPAC, have a mechanical strength proportional to their conductivity [10] [11], while the inverse is true for the graphitic host structures of GICs [12] [13]. For both GICs and IPAC, the greatest post-doped conductivity was always associated with the greatest microstructure order of the $\mathrm{sp}^{2}$ carbon host. Before doping, the best GICs were typically annealed at graphitization temperatures $\left(>2000^{\circ} \mathrm{C}\right)$ in inert atmospheres to increase the size of graphitic crystallite grain regions and $A B A B$ stacking of the graphene planes [1]. The highest conductivity IPACs, before doping, required mechanical stretching six to fifteen times their original length to sufficiently increase the microstructure alignment [11] [14]. The best CNT textiles have reached only $14 \%$ of copper's conductivity [10] and have yet to be mechanically stretched to the same degree as IPAC, despite the similarity as a high aspect ratio quasi-one-dimensional molecule. A multiwall CNT (MWCNT) film, for example, in a solvent under a tensile load can increase in length 22 to 40\% [15] [16] and SWCNT films can increase to $80 \%$ of their original length [17]. Chlorosulfonic acid (CSA) is the only known solvent for CNTs, causing them to protonate, electrostatically repel [10] and de-bundle [18]. Treating CNTs with CSA and then applying a tensile load up to now has led to a $15 \%$ length increase [19].

Recently, our group has found a general method within floating catalyst chemical vapor deposition (FCCVD) for creating relatively large yields $\left(>3 \mathrm{~g} \mathrm{day}^{-1}\right.$ ) of few-walled CNT textiles with a high degree of graphitic perfection and a sparse population distribution of diameters indicative of metallic enrichment [20]. For our so-called "high-hydrogen" CNT material (named for the relatively high hydrogen flows required to make it), there is one strikingly prominent radial breathing mode (RBM) at $152 \mathrm{~cm}^{-1}$ (under $785 \mathrm{~nm}$ excitation, indicative of a metallic chirality via Kataura plot) and a D peak substantially smaller than the G peak (relative to a typical FC-CVD-derived spectrum, figure 1). The striking prominence of the $152 \mathrm{~cm}^{-1}$ RBM indicates that the tubes giving rise to this peak are in resonance with the laser. Typical G:D ratios for high-hydrogen material averaged 45 , although values up to 120 were not uncommon. These are high values particularly in light of the $785 \mathrm{~nm}$ long Raman excitation wavelength, where G:D typically decreases with increasing Raman wavelength for graphite and high-quality CNT textiles [21]. Past research on this reactor previously generated predominantly metallic SWCNTs based on specific sulfur precursors becoming available sooner to the agglomerating catalyst [22]. We found that the highhydrogen material could be generated independent of the carbon fuel (n-butanol, toluene, or benzyl alcohol) and sulfur-based promotor (thiophene, carbon disulfide, or elemental sulfur). The general requirements were sufficiently low molar fraction of the sulfur promotor and a high hydrogen carrier gas flow rate to drive precursors faster into the reaction zone [20]. Mechanical and electrical properties of the high-hydrogen material were low, however, compared to other CNT materials (figure 1), presumably 
due to lack of microstructure alignment. We posit that a post-process alignment step would improve highhydrogen electrical and mechanical properties.

In this paper, we present a processing step that can be applied post-synthesis for the microstructure alignment and densification of our high-hydrogen material by mechanical stretching in a CSA bath. While several previous reports have discussed stretching of CNTs in CSA [19] and, in general, processing using CSA [10] [18] [23] [24] [25], this current work is noteworthy because of: 1) the extreme degree of "taffylike" mechanical stretching not seen before with CNT textiles (up to $250 \%$ of original length), accomplished exclusively with "high-hydrogen" materials; 2 ) the extrinsic enhancement of a novel CNT textile category - the high-hydrogen material. In the as-is state, high-hydrogen material has remarkable Raman spectra, although material properties such as specific conductivity and tenacity are relatively low. This is likely stemming from lack of microstructure alignment from the non-continuous, low draw speeds that highhydrogen material seems to require. Only after our alignment post-process does high-hydrogen material have a competitive specific conductivity and tenacity, as we will show. In [20], which is under review, we show that as-is high-hydrogen material has multiple indicators for predominantly metallic chiralities. While complementing, this paper does not necessarily rely on these markers; instead, we highlight highhydrogen material's notable Raman spectra and demonstrate its unique stretching in CSA. Note we dedope the CSA-assisted stretched material with an overnight vacuum bake that removes most of the residual CSA. While this treatment approximately halves the conductivity, it mitigates the complex influence of doping chemical species. After stretching and de-doping, the CNT textile's electrical conductivity is up to $45 \%$ greater than single-crystal graphite and approximately an order of magnitude higher than before stretching. We consider univariate correlations and multivariate models of conductivity as functions of as-is material parameters and CSA processing steps. The multivariate model is a heuristic, $2^{\text {nd }}$ order polynomial expression fitted to the multivariate data, useful for uncovering complex relationships potentially obscured under univariate analysis, while also enabling a predictive capability. These fitted polynomials are selected from a pool of polynomials generated by the variables representing as-is material parameters and CSA processing steps, which were selected for their optimum statistical significance using generalized regression techniques. We find that the degree of stretching is the dominant predictor for specific conductivity, although all trends eventually saturate to a limiting specific conductivity near iron and single crystal graphite. The degree of stretching is primarily predicted by the as-is mechanical properties before the CSA bath (as-is tenacity, elongation to break, and linear density). As-is Raman G':G ratio is a good marker for post-treated stretchability and conductivity. The asis upper-limit graphitic oxidation temperature in thermogravimetric analysis (TGA) is also a good indicator of post-treated stretchability and conductivity, as well as tenacity. Noteworthy, and contrary to other findings [10] [26], the as-is Raman G:D ratio has little influence on the stretched material properties; further, the tenacity is not impacted by the stretching degree.

\section{Experimental Methods}

CNT production. We generated CNT fibers via FC-CVD with a vertically-oriented mullite tube furnace, with inner diameter (ID) $65 \mathrm{~mm}$ and length $1500 \mathrm{~mm}$. Precursors were mixed into a single liquid solution and injected via a pump from the top of furnace together with preheated $\left(150^{\circ} \mathrm{C}\right)$ hydrogen gas, where the injector is a stainless steel (SS) pipe with outer diameter (OD) $1 / 4$ inch and length $135 \mathrm{~mm}$, as measured from the top-flange of the vertical mullite tube. For fuel, ferrocene powder was mixed with various carbon sources (either toluene, benzyl alcohol, and n-butanol) and sulfur compounds (either thiophene, carbon 
disulfide, or elemental sulfur) in varying concentrations (typical for high-hydrogen material: benzyl alcohol $(20 \mathrm{~mL})$, thiophene $(0.314 \mathrm{~mL})$, and ferrocene $(0.956 \mathrm{~g})$ yielding the following concentrations: $\mathrm{C}: \mathrm{S} 362.7$, $\mathrm{S}: F e$ 0.76, C:Fe 276.6 [20]). Hydrogen flow was in the range of 1 to $5 \mathrm{~L} \mathrm{~min}^{-1}$, although the high-hydrogen regime started above approximately $2.5 \mathrm{~L} \mathrm{~min}^{-1}$ and best results were typically at $5 \mathrm{~L} \mathrm{~min}^{-1}$. To produce at least a partially spinnable or continuous fiber, the fuel was metered at $20 \mu \mathrm{L} \mathrm{min}^{-1}$ for every $1 \mathrm{~L} \mathrm{~min}^{-1}$ of hydrogen. This ratio could change by $20 \%$ before complete loss of fiber continuity. This reactor had a single temperature zone set between 1270 and $1300{ }^{\circ} \mathrm{C}$, although this was typically set to $1290^{\circ} \mathrm{C}$.

At the bottom of the FC-CVD reactor was a cyclone generating compartment called a gas valve that consisted of several tangential lines of alternating nitrogen flow and suction. This gas valve enabled the CNT aerogel forming within the tube to be continually extracted as a fiber outside of the reactor, while keeping the reactive gas environments of the reactor and outside air separated. Fiber was drawn and collected onto a bobbin-roller at a linear speed of 5 to $30 \mathrm{~m} \mathrm{~min}^{-1}$ (typically $20 \mathrm{~m} \mathrm{~min}^{-1}$ ). After collecting material for between 5 and $30 \mathrm{~min}$, the resultant mat was manually compressed and densified around the roller without any condensation from solvents. This results in a smooth, opaque CNT mat with partial microstructure alignment. Further details of the FC-CVD reactor with mixed liquid fuel injection and highhydrogen material can be read here [20]; we also used another similar FC-CVD reactor to generate highhydrogen material based on mixing precursors in a vapor/gas phase, details can be read here [27] [28].

CSA-assisted stretching and vacuum bake. Now we detail the CSA-assisted stretching technique (See supplemental material for a video demonstration). The partly aligned CNT film was laser cut (SPI G3 $1 \mu \mathrm{m}$ fiber laser marker) into CNT ribbons $4 \mathrm{~mm}$ wide and typically $70 \mathrm{~mm}$ long. The ribbon thickness was variable and depended greatly on CNT reactor conditions and overall collection time; the CNT ribbons were therefore weighed to record a linear density. The CNT ribbon was placed onto the end of a scissorlike apparatus (see figure 1) made from PTFE. This end of the PTFE apparatus would soak and stretch the CNTs in a $90 \mathrm{~mm}$ wide petri dish of CSA, while the other end is manually spread apart. Mechanically clamping the CNTs to the PTFE scissor-ends was not straightforward because the CNT ends tended to slip out from any PTFE-made clamp when tension was applied in the CSA bath. We found that placing PTFE bolts through the CNT ribbon and fastening with PTFE-made nuts successfully prevented any slippage from the clamps. Typically, after threading and bolting, the CNT ribbon started as 30 to $40 \mathrm{~mm}$ long within the PTFE scissor tool. The CNT ribbon within the CSA bath was manually stretched to the greatest degree possible, as subjectively determined before any major visible tearing would occur. In many cases the edge of the $90 \mathrm{~mm}$ wide petri dish was reached before the CNT ribbon was fully stretched. After the soaking and stretching, the CNT ribbon was transferred to a chloroform bath to wash out most of the CSA (typically 0 to $100 \mathrm{~min}$ ). Next, it was transferred to an acetone bath (typically 1 to $50 \mathrm{~min}$ ) and then set out in air, where rapid evaporation of the solvent would condense the stretched ribbon. Even after drying in air, it was found that a considerable amount of acid and solvent remained trapped in the stretched material. In some cases, the wet material was rolled between a PTFE cylinder and PTFE sheet multiple times. Drying with a $300{ }^{\circ} \mathrm{C}$ vacuum oven overnight restored the material to within $10-20 \%$ of its original weight and removed any residual acid that potentially influences the conductivity haphazardly and sub-optimally.

Measurement details. The following describes the measurement procedures for our CNT materials in both the "as-is" state following production and after CSA processing.

Raman spectra were collected with an unpolarized Bruker Senterra Raman spectrometer at $785 \mathrm{~nm}$ laser excitation (20x objective, $5 \mathrm{~s}$ integration time, 5 accumulations, $10 \mathrm{~mW}$ laser power at 5 different 
measurement points on the sample). The G:D ratio was calculated by measuring integrated area under the curve, after a base line subtraction. Procedural details can be referenced here [21]. The $G^{\prime}: G$ ratio was calculated by simply dividing the peak heights, and the $\mathrm{G}^{\prime}$ position was also recorded. Scanning electron microscopy (SEM) was carried out using an FEI Nova NanoSEM at $10 \mathrm{keV}$.

Because of the large number of as-is samples, thermogravimetric analysis (TGA) of as-is material was systematically collected with a TA Q500 TGA under dynamic heating conditions and synthetic air to $1000^{\circ} \mathrm{C}$. Remarkably, with our typical dynamic heating ramp rate up to $1000^{\circ} \mathrm{C}, \mathrm{CSA}$-assisted stretched CNT material would still remain and would keep a high G:D ratio. A possible explanation is that the material is so dense that only the fiber surface is exposed to air and the graphitic perfection makes the burn at the surface relatively slow. Therefore, for those limited number of samples that were being directly compared with CSA treatment, we use a constant heating rate of $5 \mathrm{~K} \mathrm{~min}^{-1}$. TGA data considered in this paper include the amorphous carbon content (minor peak that burns typically between 300 and $450{ }^{\circ} \mathrm{C}$ ), as well as remaining residual catalyst. As for the graphitic populations in TGA, the complex and varied nature of the TGA peaks make their consistent and bias-free representation as listable metrics challenging. Developing more systematic cataloging of fitted TGA peaks is a focus of our future work. In this paper, for simplicity, we simply record the start and end temperature of all the CNT oxidation events within a sample grouped together, as determined by the TGA trace deviating from the baseline.

Linear density $\left(\mathrm{mg} \mathrm{mm}^{-1}\right)$ of laser cut ribbons was measured using a Sartorius microbalance; the resistance per millimeter $\left(\Omega \mathrm{mm}^{-1}\right)$ was measured using a four-probe Keithley multimeter and home-built test stand. Specific conductivity was calculated using $\left(\left(\mathrm{mg} \mathrm{mm}^{-1}\right) \times\left(\Omega \mathrm{mm}^{-1}\right)\right)^{-1}$. Specific conductivity, or conductivity divided by density, is a more practical metric than conductivity because it accounts for density and does not require measuring a variable cross-sectional area [24] [28]. As a measure of anisotropy [29] of the asis material, we also measured the conductivity orientation parameter, defined here as the specific conductivity measured with the spinning direction divided by the specific conductivity against the spinning direction. Tenacity $\left(\mathrm{N}\right.$ tex ${ }^{-1}$ ) of the acid stretched material was measured using a Hounsfield tensile tester

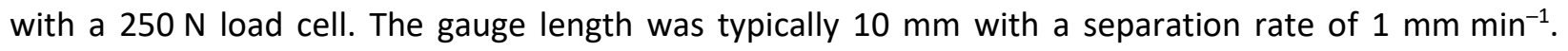
Custom-built clamps fastened the ends of the fiber as it is pulled apart, aided by tabs glued to the fiber's ends for better grip support. Tenacity $\left(\mathrm{N} \mathrm{tex}^{-1}\right)$ and elongation to break (\%) of the as-is material was measured using a Favimat tensile tester (Textechno, Germany) with a $210 \mathrm{cN}$ load cell. The percent length increase was calculated by measuring the length of the as-is CNT film/ ribbon held without slack between the PTFE clamps before CSA treatment; then again after the CSA-assisted stretching and subsequent solvent baths. The difference between the two was divided by the original, as-is length.

A database was created (see supplemental information) to keep track of the material properties after CSA-assisted stretching, as well as the associated material properties before CSA-assisted stretching (asis) and specification of the CSA-assisted stretching processing steps. The dataset is explored using JMP data analysis software [30]. We first considered univariate correlations and then consider validated, multivariate statistical models to complement. The multivariate model can uncover complex relationships potentially obscured by univariate analysis and provide a predictive capability that yields a maximum possible performance. Because of the intercorrelation between the as-is material properties, models were constructed by generalized linear regression (Estimated method: adaptive LASSO) under K-Fold or Bayesian Information Criteria (BIC). Here, a response surface is formed from the most statistically significant polynomial terms - down-selected by the generalized regression methodology from a larger pool of polynomial terms. The larger pool of $2^{\text {nd }}$ order polynomial terms are generated from the as-is 
material parameters (as-is conductivity, tenacity, elongation to break, orientation parameter, amorphous carbon percentage, graphitic burn start temperature, graphitic burn end temperature, residual catalyst, $\mathrm{G}: \mathrm{D}$ ratio, G':G ratio, and $\mathrm{G}^{\prime}$ position) and CSA processing parameters (percent length increase, CSA bathing time, chloroform bathing time, acetone bathing time, CSA's cumulative number of stretches, number of rolls in roller, and linear density). A model is judged based on its predictive power. For model validation, $25 \%$ of the dataset was randomly selected as a test set before model construction. After term selection and fitting, the measured values were compared against the model's predicted values with this unbiased test set to guard against overfitting. $R^{2}$ is a metric judging how closely a model fits the data; a higher value means a better fit, and smaller $R^{2}$ values will arise from imperfect models, unknown factors, and system noise. The adjusted $R^{2}$ penalizes the addition of an excessive number of factors into the model, which generally and arbitrarily increases $R^{2}$; the adjusted $R^{2}$ can be equal to or less than $R^{2}$. The test set $R^{2}$ is the standard $R^{2}$ generated by using data not used for fitting and model building. It is possible the model fits to noise in the system and, when the unbiased data is entered into such a model, it will result in a lower $\mathrm{R}^{2}$ than the one generated from the primary dataset.

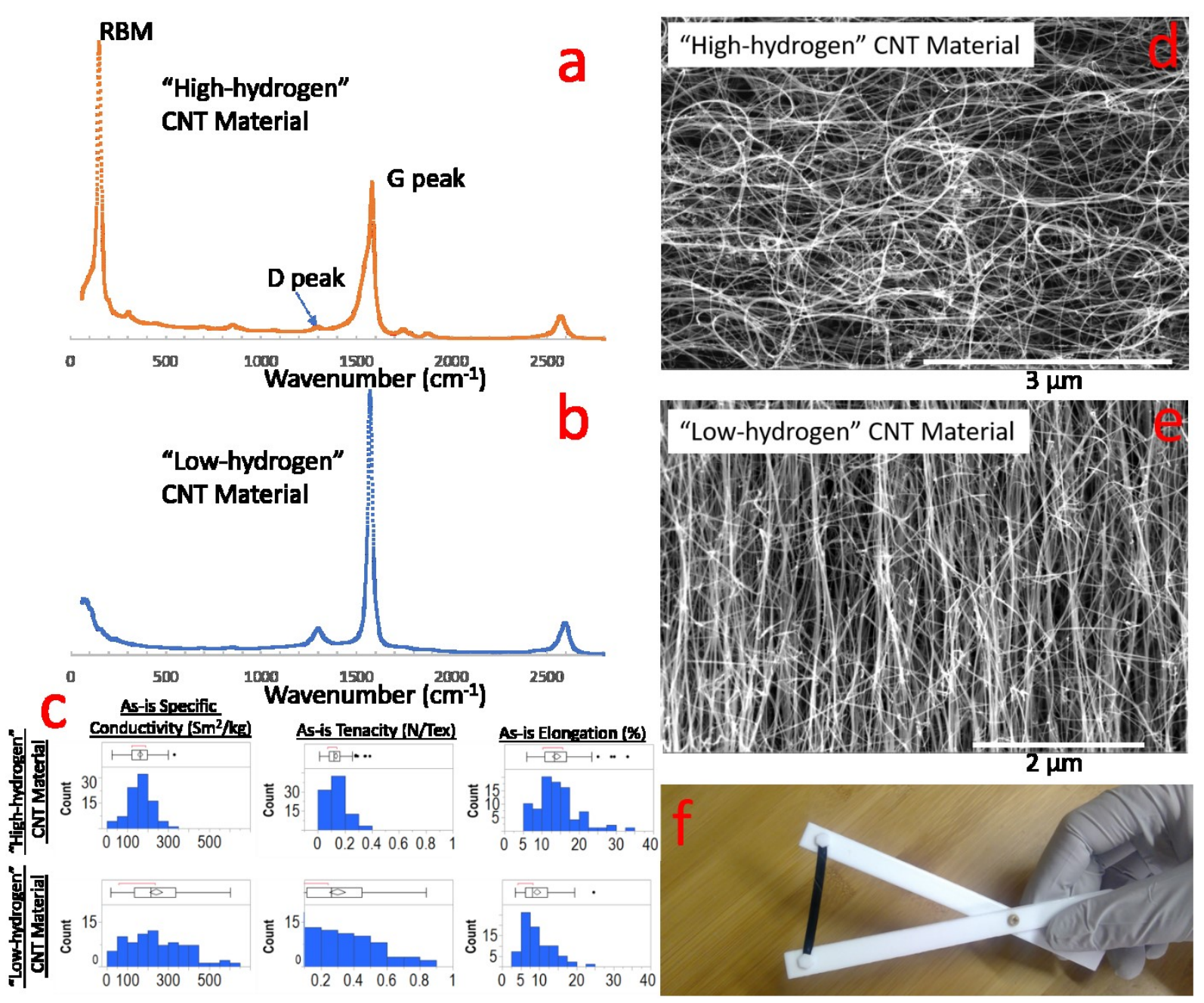

Figure 1. As-is properties of the "high-hydrogen" CNT material and more typical "low-hydrogen" CNT material. A, The as-is Raman spectra of the high-hydrogen CNT material with its prominent radial breathing mode (RBM) at $152 \mathrm{~cm}^{-1}$, followed by 
multiple overtones, and an exceedingly small D peak for $785 \mathrm{~nm}$ Raman laser excitation [21]. As explained in [20] it has signatures of metallic metallicity enrichment. B, example of a typical "low-hydrogen" Raman spectra from our FC-CVD reactors; this material stretches marginally in CSA similar to other published work [15] [16] [17] [19]. C, histograms of as-is conductivity, tenacity, and elongation-to break of both material classes. Note that the as-is material collected here is aerogel drawn on a rotating mandrill and is afterwards manually smoothed over as a mat. More sophisticated extraction involving online fiber tensioning and acetone condensation can achieve $\mathbf{2} \mathbf{N}$ tex ${ }^{-1}$ [27]. Scanning electron microscopy (SEM) photographs of d, high-hydrogen material and with e, low-hydrogen material. F, photograph of the laser-cut CNT strip held in place with PTFE bolts on a PTFE scissor tool. 

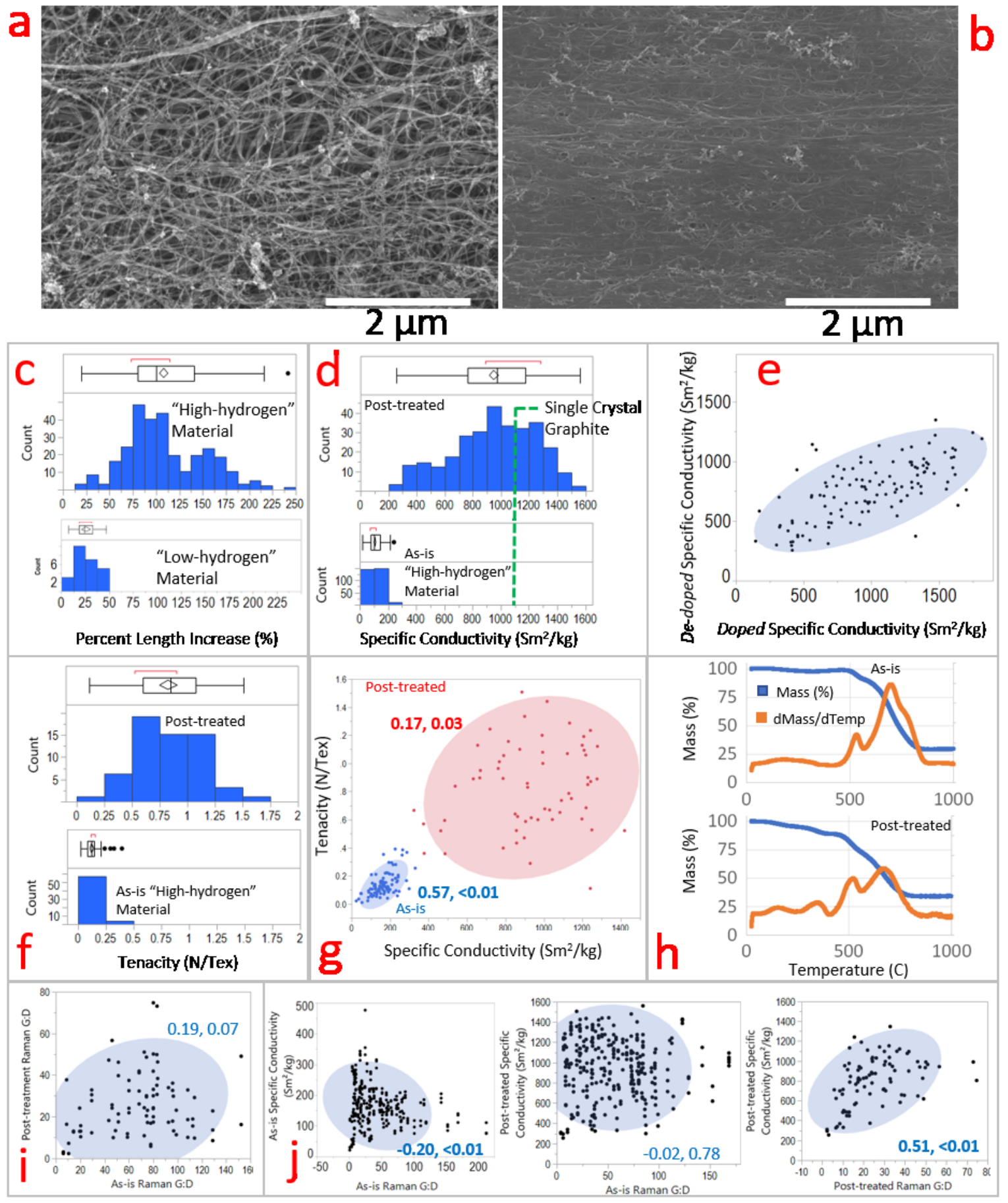

Figure 2. $a$ and b, SEM photographs of before and after the CSA-assisted stretching showing improvement in microstructure alignment and densification. $\mathrm{C}$, histograms of the percentage length increase for the high-hydrogen CNT material and lowhydrogen CNT material during CSA-assisted stretching. We find that only the high-hydrogen material stretches to an extreme degree. $D$, histograms of as-is and post-treated specific conductivity showing the magnitude change in conductivity that compares to single-crystal graphite. $E$, plot of conductivity before the vacuum oven bake versus conductivity after the vacuum oven bake. This shows the doping influence of residual CSA. F, histograms of as-is and post-treated tenacity showing substantial improvement. G, conductivity versus tenacity of as-is and CSA treated CNT material. H, TGA curves (blue) with 
mass in percentage and its derivative (orange) for as-is material and CSA-assisted stretched material. I, plot of the as-is Raman G:D ratio versus post-treated, showing degradation after CSA exposure. J, conductivity versus Raman G:D ratio between various combinations of as-is and post-treated states. Plots are also accompanied by a Pearson correlation coefficient and probability in blue (bold indicates statistical significance with probability less than 0.05 ).

\section{Results and Discussion}

Stretching degree. CNT strips were mechanically stretched in a CSA bath using a manual scissor tool made from polytetrafluoroethylene (PTFE), as described in Experimental Methods section above. After the CSAassisted stretching, samples were soaked in chloroform, followed by acetone to rinse out CSA and densify the material. In a few circumstances, they were then mechanically densified with a PTFE roller. Afterwards, all samples were vacuum baked overnight to remove any residual CSA and solvents. Samples after this vacuum bake are termed "post-treated." Figures $2 a$ and b show representative SEM micrographs of before and after the post-treatment: the post-treated CNT textiles have increased alignment and density, while bundle edges are less defined. While there are many techniques to quantify microstructure alignment, such as X-ray diffraction [31] and Fourier transform analysis of scanning electron microscopy [32], here we used established techniques with polarized Raman spectroscopy [33] [34] (see supplemental section 1 for further description and results). We found a positive relationship between the anisotropy indicated by the polarized Raman signal and the degree of stretching. For example, as-is material had a Raman anisotropy value of approximately 3 and material stretched over $150 \%$ percent could have a Raman anisotropy value up to approximately 15.

Figure $2 \mathrm{c}$ is a histogram of the percentage length change from the stretching process. We always stretched to the maximum degree possible, either to the point tearing would start or the edges of the petri dish were reached. Stretching to $100 \%$ of the original length was common with high-hydrogen material, while in some cases stretching up to $250 \%$ was possible. Typically, similar to taffy candy, high-hydrogen samples stretch with little tension required. Stretching of more-typical, low-hydrogen CNT material (with broader, lower, or no RBM populations and lower G:D ratios) required manually-applied tension and only stretched to a maximum of $50 \%$ (see histogram, Figure 2c), which is similar to other stretching results in CNT literature [15] [16] [17] [19]. Later in this paper we show that the maximum degree of stretching in CSA is related to the as-is mechanical properties, such as low tenacity and high elongation to break under tensile load in normal, dry conditions. Typically, as-is low-hydrogen CNTs have better microstructure alignment and resultantly higher as-is tenacity and elongation to break (see figure 1c) compared to as-is highhydrogen material. On first inspection, it could be argued that high-hydrogen material's uniquely large degree of stretching in CSA is simply from its lack of microstructure alignment and as-is mechanical properties. In the supplemental section 2 however, we plot the percentage length increase for stretching in CSA versus the as-is elongation to break, for both high and low-hydrogen material. As shown, there is a significant correlation for the high-hydrogen samples and none for low-hydrogen. This is also the case even when we specially produced low-hydrogen material under particularly low reactor winding or extraction speeds, yielding atypically low as-is tenacity and high elongation to break, and then stretched in CSA. We conclude that the high-hydrogen material's uniquely large degree of stretching in CSA over other CNT materials is not from some prosaic lack of microstructure alignment or as-is mechanical properties, but rather from some intrinsic feature of the high-hydrogen CNTs themselves. It was pointed out previously [10] [35] that only few-wall CNTs with a high degree of graphitic perfection protonate successfully and dissolve into CSA solution; our results with the high-hydrogen material are possibly a similar manifestation of this phenomenon, although our CNTs never disperse into a solution likely due to 
their long tangled nature. From now on our discussion will just consider the acid-stretching of highhydrogen material.

Conductivity. Figure $2 \mathrm{~d}$ shows specific conductivity histograms of both before and after the posttreatment. From the as-is state, there is an order of magnitude enhancement where now a significant portion of the specific conductivity distribution is above the specific conductivity of single-crystal graphite $\left(1100 \mathrm{Sm}^{2} \mathrm{~kg}^{-1}\right)$ and at its maximum reaches $45 \%$ higher $\left(1595 \mathrm{Sm}^{2} \mathrm{~kg}^{-1}\right)$. This de-doped conductivity is comparable to the highest reported floating catalyst derived material that is accompanied by some nondoping post-processing, namely: rollers (1700 $\mathrm{Sm}^{2} \mathrm{~kg}^{-1}$ [36]), drawing dies (1770 $\mathrm{Sm}^{2} \mathrm{~kg}^{-1}$ [37]), and lasersorting (1500 Sm $\mathrm{kg}^{-1}$ [38]). Note that the overnight vacuum baking at $300{ }^{\circ} \mathrm{C}$ is intended to remove trapped liquid and physisorbed chemical species for better material stability and repeatability. Residual acid has the side-effect of conductivity enhancement, even for predominantly metallic CNT textiles, where acids and other electron accepting chemical species aid in transport across CNT structure junctions [39] [40] [41] - beyond simply adding charge carriers. Figure 2e shows the reduction in conductivity from before the vacuum oven to after, showing the effectiveness of the de-doping step. Shifts in Raman spectra position have been used to monitor chemical doping [42] [43] or electrostatic gating [44]. When analyzing the post-treated samples, there is some correlation between the post-treated conductivity and posttreated Raman G peak position (Corr. 0.20, Prob. 0.06, see Supplemental section 3)-so some residual doping cannot be ruled out. The absolute shift in position is small however, where most (86 out of 93 measured) have post-treated $\mathrm{G}$ peak positions below $1590 \mathrm{~cm}^{-1}$, compared to other studies where substantial doping shifted $\mathrm{G}$ peak positions to $1600 \mathrm{~cm}^{-1}$ and above [42] [43]. CNT textiles are well known to self-dope by atmospheric constituents anyway, so we view our vacuum baking as a practical, though not perfect, de-doping and stabilization procedure. Over a period of five months, for example, our posttreated conductivities were stable within $5 \%$ of their initial post-treated value, on average. The fact that de-doped specific conductivity can be well above single-crystal graphite is encouraging: as shown with the GICs, the highest quality single-crystal graphite leads to the best bulk room temperature conductors (150\% that of copper) when properly doped [1] [2] [3] [4] [5] [6]. This signals that for CNTs with high dedoped specific conductivity, after proper conditioning and doping, approaching and exceeding GIC performance is not out of the question.

Tenacity. As shown in figure $2 \mathrm{f}$, the tenacity after the CSA-assisted stretching procedure is also substantially improved ( $0.85 \mathrm{~N}$ tex $^{-1}$ average, $1.6 \mathrm{~N} \mathrm{tex}^{-1}$ maximum). For purposes of comparison, note our post-treatment morphology is a long, stiff film or ribbon, and not a microfiber. Our tenacity is not as high as the highest reported CNT textiles: 2.0 [27] to 3.3 [45] to $3.7 \mathrm{~N} \mathrm{tex}^{-1}$ [46] and, with CSA posttreatment soaking, $2.19 \mathrm{~N} \mathrm{tex}^{-1}$ [47]. At present, the tenacity is closer to fibers made from extruding CSA CNT solutions into a coagulant: 1.62 [10] to $1.7 \mathrm{~N} \mathrm{tex}^{-1}$ [48]. For reference, high-strength commercial carbon fiber can be to 3.4 to $3.9 \mathrm{~N}^{-1} \mathrm{x}^{-1}$ [12] [49] [50]. Figure $2 \mathrm{~g}$ shows the relationship between tenacity and conductivity. In the as-is material, there is relatively strong correlation (Corr. 0.57, Prob. <0.01) and this is in agreement with CNT literature [10] and long-chain conductive polymers [11]; here, the implicit dominant parameter affecting both conductivity and tenacity is microstructure alignment. After the CSAassisted stretching procedure the correlation is still there (Corr. 0.17, Prob. 0.03), although weaker. This decoupling between tenacity and specific conductivity after post-treatment signals that microstructure alignment no longer simultaneously drives specific conductivity and tenacity.

TGA. Figure $2 \mathrm{~h}$ shows TGA of the as-is and CSA processed material. As shown, a high amount of residual catalyst is present in the as-is material and persists after CSA treatment ( $25-35 \%$ by weight). At best, 
residual catalyst is merely dead weight or, more likely, also impacts the intrinsic transport [24] [26]; we attempted many standard post process techniques [26] [51] [52] [53] [54] to remove the residual catalyst both before and after the CSA-assisted stretching process. This includes various combinations of oxidation (baking in air 300 to $500{ }^{\circ} \mathrm{C}$ ), hydrogen peroxide soaking, inert atmosphere annealing (up to $900{ }^{\circ} \mathrm{C}$ ), and additional acid baths ( $\mathrm{HCl}$ or additional CSA exposure) to remove first any carbonaceous layer protecting the catalyst and then the catalyst itself [51] [52] [53] [54]. So far, if purification is applied before stretching, this leads to weakened CNT material that easily breaks in CSA instead of stretches. If purification is applied after, this, so far, has led to stretched material with compromised properties. Future work is focused at successfully reducing catalyst content during the growth process itself. Correlations between other properties and residual catalyst will be discussed in the next section and will annotate some previous assumptions.

Raman. Raman G:D ratio has been a qualitative indicator for CNT's graphitic perfection or degree of amorphous carbon contamination. In high-purity situations, the G:D ratio quantitatively indicates CNT length or a characteristic length between defects [21] [55]. Figure $2 \mathrm{i}$ shows the Raman G:D ratio relationship between before and after the CSA-assisted stretching procedure. As shown, the CSA exposure lowers the Raman G:D ratio indiscriminately, where there is only weak correlation of G:D before and after CSA soaking. In other words, it could be expected that a relatively high as-is G:D ratio, while degrading in CSA, should still be relatively high amongst the post-treated samples-this correlation however, if there, is weak and at the edge of significance.

A degraded G:D ratio is the expected general side effect of powerful acids introducing defects and adding functional groups to the $s p 2$ structure [52] [56]. Figure $2 \mathrm{j}$ shows the various stages of conductivity against the various stages of G:D ratio: first, the as-is conductivity versus as-is $\mathrm{G}: \mathrm{D}$ ratio shows negative correlation (Corr. -0.20, Prob. < 0.01); second, post-treated conductivity versus as-is G:D ratio does not show any correlation (Corr. -0.02, Prob. 0.78); third, post-treated conductivity versus post-treated G:D ratio showing strong positive correlation (Corr. 0.51, Prob. < 0.01). The evolution of these correlations is noteworthy. Post-treated conductivity scaling nearly linearly with G:D ratio has been demonstrated before, under a $785 \mathrm{~nm}$ excitation, for highly aligned CNT textiles [10]. Their findings were that conductivity and $785 \mathrm{~nm}$ G:D ratio scales nearly linearly with CNT aspect ratio. Unaligned CNT film reports also demonstrated conductivity increasing with CNT length [57] [58] [59]. Therefore, the as-is material's negative correlation with G:D ratio was unexpected; we also verified it with another independent dataset of our as-is highhydrogen FC-CVD material. A possible explanation is that in less aligned material, CNT junctions have a greater impact on the transport. Here, polymeric carbons can possibly even enhance transport across junctions [28] [60], while this would tend to lower the G:D ratio. In an unaligned state, the CNT intrinsic conductivity and $\mathrm{G}: \mathrm{D}$ ratio would be less important to the conductivity. 


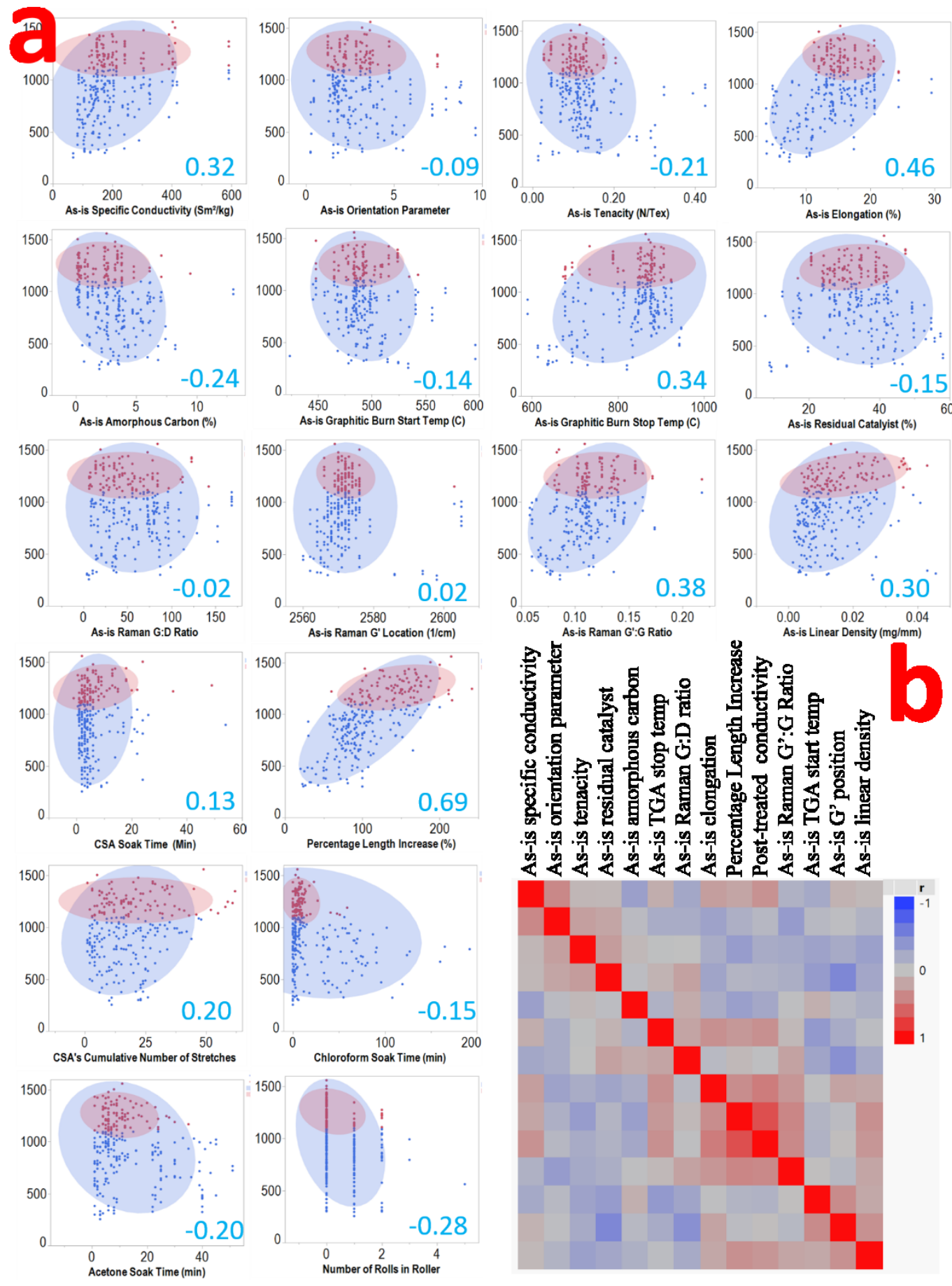

Figure 3. a, Correlation map of post-treated specific conductivity on the vertical axis against as-is material properties and the various CSA processing parameters on the horizontal axis. Blue indicates the whole dataset (with blue correlation parameters) and red indicates the subset of conductivity greater than single-crystal graphite. b, Correlation color map showing intercorrelations between as-is material properties and selected CSA processing parameters where red indicates positive correlation and blue indicates negative. Parameter columns are as indicated; parameter rows are in the same order although are omitted for clarity. 
Univariate Correlations. Figure 3a shows specific conductivity as univariate correlations with as-is material properties (as-is conductivity, tenacity, elongation to break, orientation parameter, amorphous carbon percentage, graphitic burn start temperature, graphitic burn end temperature, residual catalyst, $\mathrm{G}: D$ ratio, $\mathrm{G}^{\prime}: G$ ratio, and $\mathrm{G}^{\prime}$ position) and CSA processing parameters (percent length increase, CSA bathing time, chloroform bathing time, acetone bathing time, CSA's cumulative number of stretches, number of rolls in roller, and linear density). Blue data points and shaded regions represent the entire dataset; red data points and regions represent a high conductivity subset for specific conductivity values above $1100 \mathrm{Sm}^{2} \mathrm{~kg}^{-1}$ (the specific conductivity of single-crystal graphite). These correlations are not necessarily causal, and most parameters are partly intercorrelated between each other, meaning they cannot be controlled completely independently. Figure $3 \mathrm{~b}$ shows a color map of all the intercorrelations of as-is material properties, sorted so that similarly correlated as-is parameters are clustered together.

Post-treated specific conductivity's positive correlation to the "as-is elongation" (Corr. 0.458, Prob. $<0.001$ ) and negative correlation to "as-is tenacity" (Corr. -0.212 , Prob. 0.002) is expected from a mechanical stretching process. The positive correlation to "as-is specific conductivity" (Corr. 0.325, Prob. $<0.001$ ) is similarly expected. There is also correlation to "as-is Raman G':G ratio" (Corr. 0.381, Prob. $<0.001$ ). In contrast to the better understood G:D ratio, a universally accepted quantitative interpretation behind the $G^{\prime}: G$ ratio for CNT textiles seem to be lacking, although some literature supports that higher $\mathrm{G}^{\prime}$ intensities are linked to greater metallic SWCNT populations from stronger phonon coupling [61] [62] [63]. It has also been shown that $G^{\prime}: G$ is more sensitive to defects just on the CNT structure, where G:D is influenced by both structural defects as well as amorphous carbon [64]. Supplemental section 4 shows that the "as-is Raman G':G ratio" is also strongly linked to the stretching degree.

In terms of TGA parameters, the negative correlation between conductivity and "as-is residual catalyst" (Corr. -0.145 , Prob. 0.034 ) is not as strong as one would expect considering it comprises 25 to $40 \%$ of the weight; this will be addressed soon in the multivariate modeling. The percentage weight of "as-is amorphous carbon", taking up no more than $10 \%$ of the total weight, in fact has a stronger negative correlation (Corr. -0.241 , Prob. $<0.001$ ) than the residual catalyst. Conductivity is correlated to a lower "TGA graphitic burn start temperature" (Corr. -0.140, Prob. 0.04) and more strongly correlated with higher "TGA graphitic burn end temperature" (Corr. 0.338, Prob. <0.001). Supplemental section 4 and 5 shows that "TGA graphitic burn end temperature" is also positively correlated with the CSA stretchability and the post-process tenacity. In the intercorrelation map (figure $3 b$ ), there is intercorrelation between the two as-is TGA parameters (Corr. -0.34 Prob. $<0.001$ ) where a higher ending burn temperature tends to have an earlier starting burn temperature. It has been shown that residual catalyst lowers the initial oxidation temperature of SWCNTs [54] and that production of SWCNTs tend to have greater percentage residual catalyst than larger CNTs [51]. Again from the intercorrelation map, indeed, there is negative correlation (Corr. -0.348, Prob. <0.001) between "as-is residual catalyst" and "TGA graphitic burn start temperature." Further, there is intercorrelation between "as-is residual catalyst" and "as-is Raman G:D ratio" (Corr. 0.23, Prob. $<0.001$, see figure 3b). A narrative deserving of further exploration is that higher "TGA graphitic burn end temperatures" are associated with better quality CNTs and better stretched bulk material properties, although this is offset by more residual catalyst production and this lowers the "TGA graphitic burn start temperature." 
With respect to CSA processing parameters, the "percentage length increase" is by far the strongest correlation (Corr. 0.691, Prob. $<0.001$ ) to post-treated specific conductivity and this illustrates the criticality of microstructure alignment and densification. Shown in supplemental section 4 , the "percentage length increase" itself is correlated with as-is mechanical properties of high as-is elongation and low as-is tenacity; as well as as-is TGA parameters of low "as-is amorphous carbon" and high "TGA graphitic burn end temperature"; as well as as-is Raman parameters of as-is Raman G':G ratio.

Between other CSA processing parameters and post-treated specific conductivity, there is positive correlation with "as-is linear density" (Corr. 0.303, Prob. <0.001) and this bodes well for scale-up. There is an unexpected positive correlation (Corr. 0.199, 0.003) with the "cumulative number of dips in the CSA bath," which is cumulative across many samples and is, in effect, the age of the CSA bath. The CSA bath noticeably ages over use, turning from clear to golden brown over many sample dips. In some samples we incorporated an extra densification step where solvent soaked material was manually-pressed between a PTFE roller and slab before the vacuum oven. While other papers reported enhanced conductivity with compression in a rolling mill [45] [65], we found the opposite (Corr. -0.282, Prob. <0.001). The stretched, solvent soaked fiber irregularly widens after rolling and this degrades the microstructure. In regard to soak time, we found that longer CSA soaking (Corr. 0.132, Prob. 0.054) and shorter solvent soaking (acetone Corr. -0.199, Prob. 0.003; chloroform Corr. -0.153, Prob. 0.026) link to higher conductivities. Recorded parameters that did not find any significant correlation were: "as-is Raman G' position" (Corr. 0.02 Prob. 0.76), "as-is Raman G:D ratio" (Corr. -0.020, Prob. 0.780), and "as-is orientation parameter" (Corr. -0.090, Prob. 0.190).

Now we examine how all correlations change when only a high conductivity subset is considered $\left(>1100 \mathrm{Sm}^{2} \mathrm{~kg}^{-1}\right)$. As shown in figure 3 (red points and shaded region), as-is material properties remarkably are no longer correlated with post-treated conductivity. A few CSA processing steps are still positively correlated: the "percentage length increase" impact has approximately halved in strength (Corr. 0.333, Prob. 0.02); the "as-is linear density" (Corr. 0.370, Prob. <0.001) and "bathing time in CSA" (Corr. 0.220, Prob. $<0.048$ ) are slightly stronger than before. The fact that, within the high conductivity subset, most correlations have vanished, and others diminished, suggests that a maximum undoped conductivity is being reached.

Supplemental section 5 shows the univariate correlations with post-treated tenacity. Similar to posttreated specific conductivity, there is a correlation with post-treated tenacity for both "as-is TGA graphitic burn end temperature" and "as-is specific conductivity." Contrasting post-treated specific conductivity, however, post-treated tenacity is negatively correlated with "as-is linear density." Notably, there is no correlation between post-treated tenacity and "percent length increase." These differences explain the weaker correlation between specific conductivity and tenacity after treatment, as illustrated earlier. Overall the tenacity/ conductivity relationship is now more closely resembling commercial carbon fiber, where the highest tensile strength requires a degree of disorder and crosslinking; more ordered commercial carbon fiber is less strong, although more conductive and stiffer [12]. 
Multivariate models. Now we build a multivariate statistical model on specific conductivity based on the as-is material properties and CSA processing steps. As-is material properties are heavily inter-corelated; statistical multivariate modeling will help determine what parameters are most important. It will also enable prediction of post-treated conductivity as functions of as-is material properties and processing steps, to include a maximum conductivity.

The "percent length increase" is the dominant univariate correlation to specific conductivity; as a model baseline, figure $4 \mathrm{a}$ is a simple fit to the $2^{\text {nd }}$ order polynomial expression generated just from the "percent length increase" parameter. While noisy, the model shows specific conductivity first linearly increasing with "percent length increase" and then saturates at $1300 \mathrm{Sm}^{2} \mathrm{~kg}^{-1}$ near the conductivities of single-crystal graphite and iron. The $R^{2}$ and adjusted $R^{2}$ are $53 \%$ and $52 \%$, the $R^{2}$ of the test set is $44 \%$. Used for model validation, the test set is randomly selected data withheld from the fitting routines. Only after the model is fitted is this test set applied to the model. Lower $R^{2}$ values here indicate the degree of overfitting. 


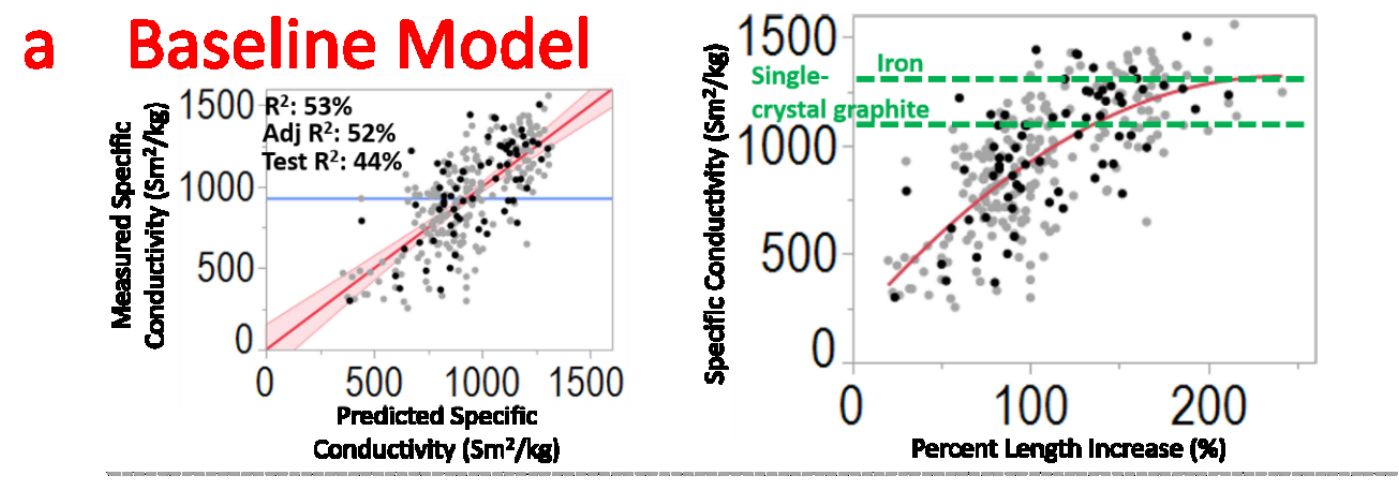

b Multivariate Model

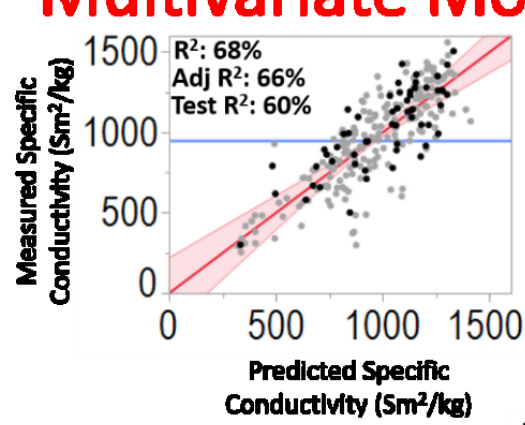

Parameter Ranking

Percentage Length Increase (\%)

As-is Elongation (\%)

Number of PTFE Rolls

As-is Specific Conductivity $\left(5 \mathrm{~m}^{2} / \mathrm{kg}\right)$

Chloroform Soak Time (mln)

As-is Residual Catalyst (\%)

As-is Linear Density $(\mathrm{mg} / \mathrm{mm})$

As-is Raman G' Position ( $\left.\mathrm{cm}^{-1}\right)$

As-is Tenaclty (N/Tex)

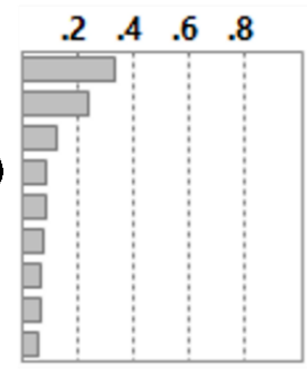

Conductlvity $\left(5 \mathrm{~m}^{2} / \mathrm{kg}\right)$
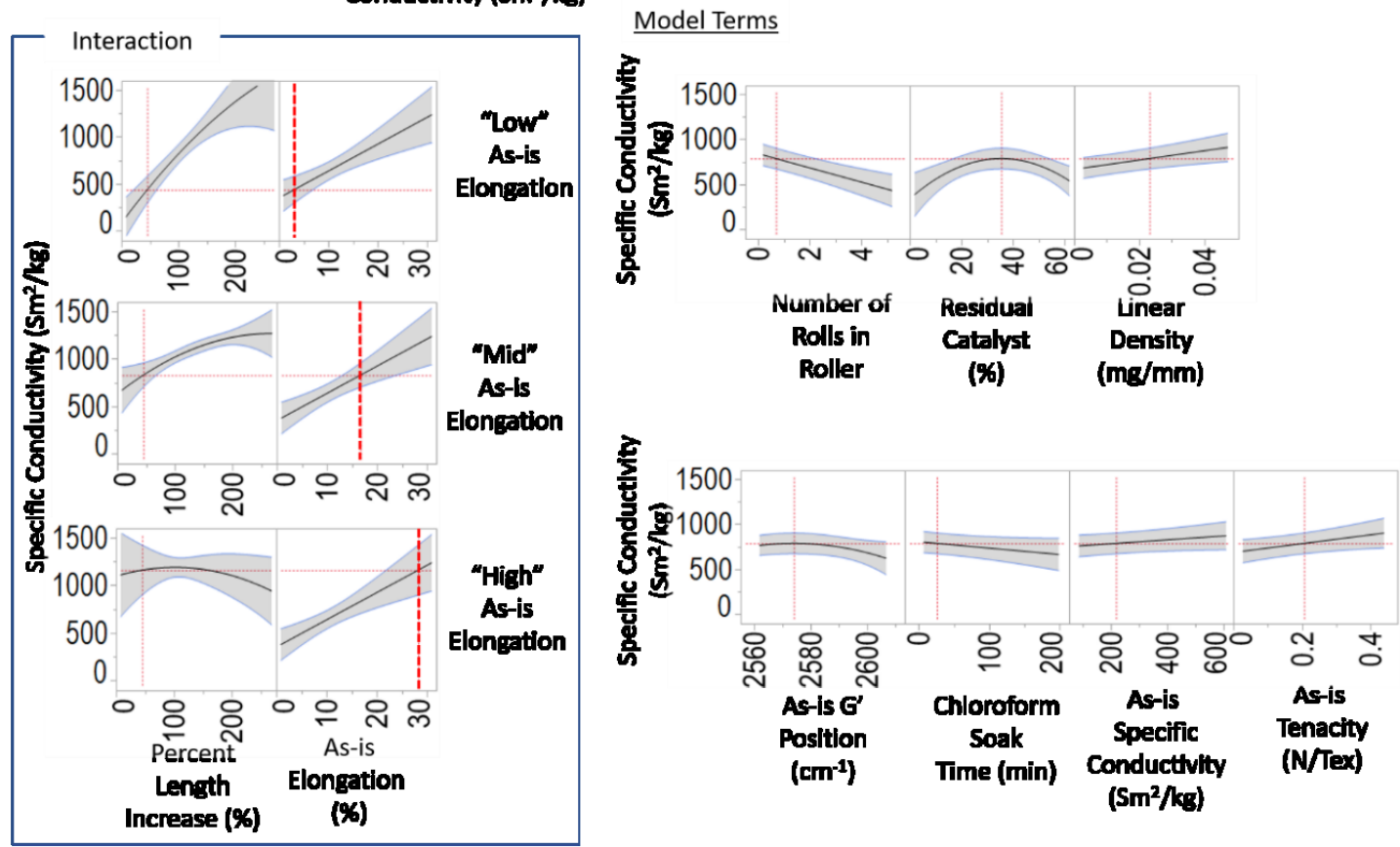

Figure 4. Two models of specific conductivity: $a$, the baseline model and, b, the multivariate model with better predictive capability. Each model has plots of predicted versus measured conductivities (to judge model quality), as well as plots of the model's polynomial terms. Grey dots indicate the data used for model fitting and black dots indicate the independent test dataset, which evaluates the model without bias after fitting. The multivariate model ranks parameters in order of importance and has an interaction term between "percent length increase" and "as-is" elongation. Because of the interaction, the exact behavior of the "percent length increase" plot depends on the value of "as-is elongation" (as indicated by the dotted red line). 
A more complex model is shown in figure $4 \mathrm{~b}$. This is a fitted second order polynomial expression generated from nine parameters, down-selected from the pool of 18 total parameters considered by the Generalized Regression with/ Adaptive LASSO routine under Bayesian Information Criteria (BIC). Figure 4 shows the actual measured conductivities plotted against the model's predicted values; black points indicate the test set. Superior to the baseline model, the $\mathrm{R}^{2}$ and adjusted $\mathrm{R}^{2}$ are $68 \%$ and $66 \%$, the test set $R^{2}$ is $60 \%$. More intricate models with better $R^{2}$ were considered, although generally resulted in inferior $\mathrm{R}^{2}$ for test set, indicating overfitting.

Many of this model's displayed trends are similar to the univariate analysis. Figure $4 \mathrm{~b}$ shows the parameter importance ranking. "Percent length increase" and "elongation" are the strongest predictors, followed by smaller contributions from "number of rolls in roller", "as-is conductivity", "chloroform soak time", "linear density", and "as-is tenacity" as earlier indicated by the univariate correlations. Contrasting the univariate correlations, "as-is Raman G' position" is now relevant where high wavenumber values negatively impact conductivity. In general the $\mathrm{G}^{\prime}$ position is approximately twice the wavenumber as the D peak, although in CNTs its exact position is influenced by structural factors such as diameter (smaller diameters shift to lower wavenumbers) and number of walls [62] [66]. Another important contrast to the univariate analysis is the interaction between "percent length increase" and "as-is elongation". Figure 4b shows a low "as-is elongation" configuration and a high "as-is elongation" configuration. When "as-is elongation" is relatively low and the material is most difficult to stretch, greater "percent length increase" leads to greater conductivity. As "as-is elongation" increases and makes stretching easier, this trend subsides and eventually reverses. Another contrast with the model is "as-is residual catalyst," where there is a non-monotonic response with a maximum at $33 \%$. This explains why the univariate correlation was weak, although it is unexpected a maximum exists for a parameter considered dead weight. We consider two possible explanations: 1 ) the residual catalyst adds to the transport. The specific conductivity of iron $\left(1320 \mathrm{Sm}^{2} \mathrm{~kg}^{-1}\right)$ is near the value of single-crystal graphite $\left(1100 \mathrm{Sm}^{2} \mathrm{~kg}^{-1}\right)$ and our best conductivity values saturate between these two (figure 3a). The residual catalyst however is known to be various iron carbides and oxides, typically encapsulated by amorphous or graphitic carbon shells [51] [52] [53] [54]; it is not clear to what degree iron carbon connections could yield a competing percolation path comparable to the graphitic structure itself. 2) Earlier we discussed how high-quality CNTs are linked to greater residual catalyst; it is possible this interplay results in a complex conductivity response.

Restricting to values available in this study, the model's maximum predicted conductivity is $2100 \mathrm{Sm}^{2} \mathrm{~kg}^{-1}$. The baseline model's conductivity saturated at $1300 \mathrm{Sm}^{2} \mathrm{~kg}^{-1}$. It is worth considering that the process and high-hydrogen material is approaching an undoped, maximum threshold near these estimates. This is above single-crystal graphite $\left(1100 \mathrm{Sm}^{2} \mathrm{~kg}^{-1}\right)$, making an optimized stretched high-hydrogen CNT fiber a compelling graphitic host for intercalation doping. That is because, for graphitic intercalation compounds, approaching and exceeding copper's conductivity was based on having the highest host graphite conductivity. Simultaneously, these models' estimates are not substantially beyond single-crystal graphite. Single-crystal graphite is a square millimeter-sized, near-perfect ABAB layered stack of graphene. On an intrinsic scale, for both graphene [67] and metallic SWCNTs [68], the room temperature mean free path is approximately $1 \mu \mathrm{m}$ and limited by phonons; so even with the best extrinsic order and alignment we do not expect undoped metallic SWCNT conductivity substantially greater than undoped graphite. Further supporting this assessment, SWCNT bundles are natural, aligned and highly oriented agglomerations of SWCNTs, making them the best tubular analogs to single-crystal graphite. SWCNT 
bundles experimentally have a conductivity of 1-3 $\mathrm{MSm}^{-1}$ [40] [69], matching single-crystal graphite's conductivity at $2.5 \mathrm{MSm}^{-1}$.

\section{Conclusions}

In this report, we have demonstrated a post-synthesis processing treatment for CNT fibers that categorically increases their density and microstructural alignment, leading to substantial increases in electrical conductivity and strength, and which seems to work uniquely for a novel material class of CNT textile - the high hydrogen material. The taffy-like degree of stretching is unprecedented in CNT textiles. In order to systematically optimize properties, we presented correlation tables and validated statistical models as functions of as-is material properties and CSA processing steps. For conductivity, the degree of stretching is the dominant factor. Not all high-hydrogen samples stretch to the same degree in CSA and this was largely dependent on as-is mechanical properties. High as-is $G^{\prime}: G$ ratio is particularly correlated with a high degree of stretchability and conductivity; this metric has been linked to greater metallicity. The Raman G:D ratio, which is more well known to be correlated with high conductivity, only showed a significant positive correlation when considering the post-treated G:D ratio with post-treated conductivity. The upper limit oxidation temperature in TGA has also been a good predictor for conductivity, stretchability, and tenacity; in [20] this metric is linked to a greater degree of CNTs associated with the prominent, metallic RBM at $152 \mathrm{~cm}^{-1}$.

Correlation tables and statistical modeling indicate that the conductivity is approaching a maximum limiting threshold near that of single-crystal graphite and iron in the undoped state. To the degree that metallic SWCNTs are simply tubular analogs of graphene, with similar carrier densities and mean free paths, we do not expect great differences between the two when closely connected as an interacting ensemble (e.g. CNT cables and graphite). A CNT cable, however, is intrinsically more flexible and easier to work with than single-crystal graphite and may respond more favorably to doping once a threshold is reached (particularly for the semi-conducting SWCNTs). SWCNTs with diameter approximately $1.3 \mathrm{~nm}$ and double-wall CNTs at approximately $2.5 \mathrm{~nm}$ are as dense as graphite [70], and so a greater degree of conduction paths may be obtained relative to graphite for a well aligned assembly of smaller CNT diameters. Before these fundamental questions are answered however, the residual catalyst must be extracted from the system without disrupting the graphitic perfection or microstructure alignment. 


\section{Conflict of Interest}

No author has a conflict of interest with this research.

\section{Acknowledgements}

We acknowledge the Engineering and Physical Sciences Research Council (EPSRC) grant, "Instrumentation and Control of Carbon Nanotube Fibre Manufacture" reference number EP/M02086X/1. We also acknowledge the EPSRC Advanced Nanotube Application and Manufacturing Initiative (ANAM), reference number EP/M015211/1.

\section{References}

[1] Inagaki M. Applications of graphite intercalation compounds. J. Mater. Res. 1989; 4(6):1560-1568.

[2] Foley G, Zeller C, Falardeau E, Vogel F. Room Temperature Electrical Conductivity Of A Highly Two Dimensional Synthetic Metal: AsF $F_{5}$-Graphite. Solid State Communications 1977; 24: 371-375.

[3] Vogel F. The electrical conductivity of graphite intercalated with superacid fluroides: experiments with antimony pentafluoride. Journal of Materials Science 1977; 12: 982-986.

[4] Fischer J, Thompson T. Graphite Intercalation Compounds. Physics Today 1978; 31(7): 36

[5] Lee W, Davls G, Vogel F. Electrical Resistivity And Magnetoresistance Of Carbon/Graphite Fibers Intercalated With Nitric Acid And Arsenic Pentafluoride. Carbon 1985; 23(6): 731-737.

[6] Shioya J, Matsubara H, Murakami S. Properties OF AsF Intercalated Vapor Grown Graphite. Synth. Met. 1986; 14: 113-123.

[7] Basescu N, Liu Z, Moses D, Heeger A. High electrical Conductivity in Doped Polyacetylene. Nature 1987; 327(4):403.

[8] Kaiser A. Systematic Conductivity Behavior in Conducting Polymers: Effects of Heterogeneous Disorder. Adv. Mater. 2001; 13: 927.

[9] Currents in Plastics. Scientific American, September 1987.

[10] Tsentalovich D, Headrick R, Mirri F, Hao J, Behabtu N, Young C, et al. Influence of Carbon Nanotube Characteristics on Macroscopic Fiber Properties. ACS Appl. Mater. Interfaces 2017; 9: 3618936198. 
[11] Cao Y, Smith P, Heeger A. Mechanical and electrical properties of polyacetylene films oriented by tensile drawing. Polymer 1991; 32(7): 1210

[12] Newcomb B. Processing, structure, and properties of carbon fibers C.omposites: Part A 2016; 91: 262-282.

[13] Murday J, Dominguez D. An assessment of graphitized carbon fiber use for electrical power transmission. Synth. Met. 1984; 9: 397-424.

[14] Tsukamoto J. Recent advances in highly conductive polyacetylene. Adv. Phys.1992; 41(6):509-546.

[15] Cimpoiasu E, Sandu V, Levin G, Simpson A, Lashmore D. Angular magneto-resistance of streched carbon nanotube sheets. J. Appl. Phys. 2012; 111: 123721.

[16] Li S, Park J, Liang Z, Siegrist T, Liu T, Zhang M, et al. In situ characterization of structural changes and the fraction of aligned carbon nanotube networks produced by stretching. Carbon 2012; 50: 3859-3867.

[17] Badaire S, Pichot V, Poulin C, Launois P, Vavro J, Guthy C, et al. Correlation of properties with preferred orientation in coagulated and stretch-aligned single-wall carbon nanotubes. Journal of Applied Physics 2004; 96(12): 7509.

[18] Hecht D, Heintz A, Lee R, Hu L, Moore B, Cucksey C, et al. High conductivity transparent carbon nanotube films deposited from superacid. Nanotechnology 2011; 22: 075201.

[19] Cho H, Lee H, Oh E, Lee S, Park J, Park H, et al. Hierarchical structure of carbon nanotube fibers, and the change of structure during densification by wet stretching. Carbon 2018; 136: 409-416.

[20] Kaniyoor A, Bulmer J, Gspann T, Mizen J, Ryley J, Kiley P, et al. The Jetting Paradigm - a unique approach to high throughput production of single-wall carbon nanotube fibres. To appear.

[21] Bulmer J, Gspann T, Barnard J, Elliott J. Chirality-independent characteristic crystal length in carbon nanotube textiles measured by Raman spectroscopy. Carbon 2017; 115: 672-680.

[22] Sundaram R, Koziol K, Windle A. Continuous Direct Spinning of Fibers of Single-Walled Carbon Nanotubes with Metallic Chirality. Advanced Materials 2011; 23(43): 5064-5068.

[23] Behabtu N, Young C, Tsentalovich D, Kleinerman O, Wang X, Ma AW, et al. Strong, Light, Multifunctional Fibers of Carbon Nanotubes with Ultrahigh Conductivity. Science 2013; 339: 182.

[24] Bucossi A, Cress C, Schauerman C, Rossi J, Puchades I, Landi B. Enhanced Electrical Conductivity in Extruded Single-Wall Carbon Nanotube Wires from Modified Coagulation Parameters and Mechanical Processing. ACS Appl. Mater. Interfaces 2015; 7: 27299-27305.

[25] Zhang Q, Li K, Fan Q, Xia X, Zhang N, Xiao Z, et al. Performance improvement of continuous carbon nanotube fibers by acid treatment. Chin. Phys. B 2017; 26(2): 028802. 
[26] Zhao Y, Wei J, Vajtai R, Ajayan P, Barrera E. Iodine doped carbon nanotube cables exceeding specific electrical conductivity of metals. Sci. Rep. 2011; 1(83).

[27] Gspann T, Smail F, Windle A. Spinning of carbon nanotube fibres using the floating catalyst high temperature route: purity issues and the critical role of sulphur. Faraday discussions 2014; 173.

[28] Gspann T, Juckes S, Niven J, Johnson M, Elliott J, White M, et al. High thermal conductivities of carbon nanotube films and micro-fibres and their dependence on morphology. Carbon 2017; 114: 160-168.

[29] Li S, Park J, Liang Z, Siegrist T, Liu T, Zhang M, et al. In situ characterization of structural changes and the fraction of aligned carbon nanotube networks produced by stretching. Carbon 2012; 50: 3859-3867.

[30] "JMP Pro 13.1.0," SAS Institute , 2016.

[31] Gbordzoe S, Yarmolenko S, Hsieh Y, Adusei P, Alvarez N, Fialkova S, et al. Three-dimensional texture analysis of aligned carbon nanotube structures. Carbon 2017; 121: 591-601.

[32] Gspann T, Juckes S, Niven J, Johnson M, Elliott J, White M, et al. High thermal conductivities of carbon nanotube films and micro-fibres and their dependence on morphology. Carbon 2017; 114: 160-168.

[33] Duesberg G, Loa I, Burghard M, Syassen K, Roth S. Polarized Raman Spectroscopy on Isolated Single-Wall Carbon Nanotubes. Physical Review Letters 2000; 85: 5436.

[34] Gommans H, Alldredge J, Tashiro H, Park J, Magnuson J, Rinzler A. Fibers of aligned single-walled carbon nanotubes: Polarized Raman spectroscopy. Journal of Applied Physics 2000; 88: 2509.

[35] Parra-Vasquez A, Behabto N, Green M, Pint C, Young C, et al. Spontaneous Dissolution of Ultralong Single- and Multiwalled Carbon Nanotubes. ACS Nano 2010; 4(7): 3969-3978.

[36] Wang J, Luo X, Wu T, Chen Y. High-strength carbon nanotube fibre-like ribbon with high ductility and high electrical conductivity. Nature Communications 2014; 5: 1.

[37] Guo F, Li C, Wei J, Xu R, Zhang Z, Cui X, et al. Fabrication of highly conductive carbon nanotube fibers for electrical application. Mater. Res. Express 2015; 2: 095604.

[38] Bulmer J, Gspann T, Orozco F, Sparkes M, Koerner H, Di Bernardo A, et al. Photonic Sorting of Aligned, Crystalline Carbon Nanotube Textiles. Scientific Reports 2017; 7: 12977.

[39] Blackburn J, Barnes T, Beard M, Kim Y, Tenent R, McDonald T, et al. Transparent conductive singlewalled carbon nanotube networks with precisely tunable ratios of semiconducting and metallic nanotubes. ACS Nano 2008; 2(6): 1266-1274.

[40] Nirmalraj P, Lyons P, De S, Coleman J, Boland J. Electrical connectivity in single-walled carbon nanotube networks. Nano Lett. 2009; 9(11): 3890-3895. 
[41] Znidarsic A, Kaskela A, Laiho P, Gaberscek M, Ohno Y, Nasibulin A. Spatially Resolved Transport Properties of Pristine and Doped Single-Walled Carbon Nanotube Networks. J. Phys. Chem. C 2013; 117: 13324-13330.

[42] Skakalova V, Kaiser A, Dettlaff-Weglikowska U, Hrnarikova K, Roth S. Effect of Chemical Treatment on Electrical Conductivity, Infrared Absorption, and Raman Spectra of Single-Walled Carbon Nanotubes. J. Phys. Chem. B 2005; 109: 7174-7181.

[43] Rao A, Eklund P, Bandow S, Thess A, Smalley R. Evidence forcharge transfer in doped carbon nanotube bundles from Raman scattering. Nature 1997; 388: 257.

[44] Tsang J, Freitag M, Perebeinos V, Liu J, Avouris P. Doping and phonon renormalization in Carbon Nanotubes. Nature Nanotechnology 2007; 2: 725.

[45] Wang J, Luo X, Wu T, Chen Y. High-strength carbon nanotube fibre-like ribbon with high ductility and high electrical conductivity. Nat. Commun. 2014; 5: 3848.

[46] Park O, Choi $\mathrm{H}$, Jeong $\mathrm{H}$, Jung $\mathrm{Y}, \mathrm{Yu}$ J, et al. High-modulus and strength carbon nanotube fibers using molecular cross-linking. Carbon 2017; 118: 413-421.

[47] Cho H, Lee H, Oh E, Lee S, Park J, et al. Hierarchical structure of carbon nanotube fibers, and the change of structure during densification by wet stretching. Carbon 2018; 136: 409-416.

[48] Headrick R, Tsentalovich D, Berdegue J, Bengio E, Liberman L, Kleinerman O, et al. StructureProperty Relations in Carbon Nanotube Fibers by Downscaling Solution Processing. Adv. Mater. 2018; 30: 1704482.

[49] "Teijin," [Online]. Available: https://www.teijincarbon.com/products/tenaxr-carbon-fiber/tenaxrfilament-yarn.

[50] "Toray," [Online]. Available: http://www.torayca.com/en/download/pdf/torayca_t1100g.pdf.

[51] Hou P, Liu C, Cheng H. Purification of carbon nanotubes. Carbon 2008; 46: 2003-2025.

[52] Morelos-Gómez A, Fujishige M, Vega-Díaz S, Ito I, Fukuyo T, Cruz-Silva R, et al. High electrical conductivity of double-walled carbon nanotube fibers by hydrogen peroxide treatments. J. Mater. Chem. A 2016; 4: 74-82.

[53] Tran T, Headrick R, Bengio E, Myint S, Khoshnevis H, Jamali V, et al. Purification and Dissolution of Carbon Nanotube Fibers Spun from the Floating Catalyst Method. ACS Appl. Mater. Interfaces 2017; 9: 37112-37119.

[54] Chiang I, Brinson B, Smalley R, Margrave J, Hauge R. Purification and Characterization of SingleWall Carbon Nanotubes. J. Phys. Chem. B 2001; 105: 1157-1161.

[55] Cou S, Son H, Kong J, Jorio A, Saito R, et al. Length characterization of DNA wrapped carbon nanotubes using Raman spectroscopy. Appl. Phys. Lett. 2007; 90: 131109. 
[56] Zhou Y, Shimada S, Saito T, Azumi R. Understanding the doping effects on the structural and electrical properties of ultrathin carbon nanotube networks. J. Appl. Phys.2015; 118: 215305.

[57] Sakurai S, Kamada F, Futaba D, Yumura M, Hata K. Influence of lengths of millimeter-scale singlewalled carbon nanotube on electrical and mechanical properties of buckypaper. Nanoscale Research Letters 2013; 8: 546.

[58] Hecht D, Hu L, Grüner G. Conductivity scaling with bundle length and diameter in single walled carbon nanotube networks. Appl. Phys. Lett 2006; 89: 133112.

[59] Pereira L, Rocha C, Latgé A, Coleman J, Ferreira M. Upper bound for the conductivity of nanotube networks. Appl. Phys. Lett. 2009; 95: 123106.

[60] Thiagarajan V, Wang X, Bradford P, Zhu Y, Yuan F. Stabilizing carbon nanotube yarns using chemical vapor infiltration. Composites Science and Technology 2014; 90: 82-87.

[61] Hirschmann T, Muramatsu H, Seifert M, Wurstbauer U, et al. G' band in double- and triple-walled carbon nanotubes: A Raman study. Physical Review B 2015; 91: 075402.

[62] Kim K, Park J, Kim S, Geng H, Hyeok K, et al. Dependence of Raman spectra G' band intensity on metallicity of single-wall carbon nanotubes. Physical Review B 2007; 76: 205426.

[63] Rao R, Reppert J, Podila R, Zhang X, Rao A, Talapatra S, Maruyama B. Double resonance Raman study of disorder in CVD-grown single-walled carbon nanotubes. Carbon 2011; 49(4): 1318-1325.

[64] DiLeo R, Landi B, Raffaelle R. Purity assessment of multiwalled carbon nanotubes by Raman spectroscopy. Journal of Applied Physics 2007; 101: 064307.

[65] Han B, Xue X, Xu Y, Zhao Z, Guo E, Liu C. Preparation of carbon nanotube film with high alignment and elevated density. Carbon 2017; 122: 496-503.

[66] Lakoubovskii K, Ueno T, Kazaoui S, Kataura H. Optical Characterization of Double-Wall Carbon Nanotubes: Evidence for Inner Tube Shielding. J. Phys. Chem. C 2008; 112: 11194-11198.

[67] Wang L, Meric I, Huang P, Gao Q, Gao Y, Tran H, et al. One-Dimensional Electrical Contact to a Two-Dimensional Material. Science 2013; 342: 614.

[68] Purewal M, Hong H, Ravi A, Chandra B, Hone J, Kim P. Scaling of resistance and electron mean free path of single-walled carbon nanotubes. Phys. Rev. Lett. 2007; 98: 186808.

[69] Fischer J, Dai H, Thess A, Lee R, Hanjani N, Dehaas D, et al. Metallic resistivity in crystalline ropes of single-wall carbon nanotubes. Phys. Rev. B 1997; 55(8): R4921.

[70] Laurent C, Flahaut E, Peigney A. The weight and density of carbon nanotubes versus the number of walls and diameter. Carbon 2010; 48(10):2994-2996. 